\title{
The role of down staging treatment in the management of locally advanced intrahepatic cholangiocarcinoma: Review of literature and pooled analysis
}

\begin{abstract}
Sivesh Kamarajah ${ }^{1}$, Francesco Giovinazzo ${ }^{1}$, Keith J. Roberts ${ }^{1}$,
Pankaj Punia ${ }^{2}$, Robert P. Sutcliffe ${ }^{1}$, Ravi Marudanayagam ${ }^{1}$, Nikolaos Chatzizacharias ${ }^{1}$, John Isaac ${ }^{1}$, Darius F. Mirza ${ }^{1}$, Paolo Muiesan ${ }^{1}$, and Bobby VM Dasari ${ }^{1}$

Departments of ${ }^{1}$ Hepatobiliary and Pancreatic Surgery, and ${ }^{2}$ Oncology, Queen Elizabeth Hospital, Birmingham, UK

Backgrounds/Aims: Approximately $60-80 \%$ of patients with intrahepatic cholangiocarcinoma (iCCA) are not suitable for surgical resection due to advanced disease at presentation. This review assesses the role of surgical resection followed by down staging treatment in the management of patients with locally advanced iCCA. Methods: A systematic review and pooled analysis were performed of the relevant published studies published between January 2000-December 2018. The primary outcome measure was overall survival. Secondary outcome measures were rates of clinical benefit, margin-negative (R0) resections, overall and surgery-specific complications, and post-operative mortality. Results: Eighteen cohort studies with 1880 patients were included in the review. The median overall survival in all patients was 14 months (range, 7-18 months). Patients undergoing resection following down staging had significantly longer survival than those who did not (median: 29 vs. 12 months, $p<0.001$ ). The Clinical Benefit Rate with this strategy (complete response+partial response+stable disease) was 64\% (244/383), ranging from 33-90\%. Thirty-eight percent of the patients underwent resections with a $60 \%$ R0 resection rate and $6 \%$ postoperative mortality. Conclusions: Although the evidence to support the benefits of NAT for iCCA is limited, the review supports the use of down staging treatment and also surgical resection in the cohort with response to NAT in order to improve long-term survival in patients with locally advanced iCCA. (Ann Hepatobiliary Pancreat Surg 2020;24:6-16)
\end{abstract}

Key Words: Intrahepatic; Cholangiocarcinoma; Locally advanced; Down staging; Surgery

\section{INTRODUCTION}

The incidence of intrahepatic cholangiocarcinoma (iCCA) is about 0.7 cases per 100,000 adults in the USA. ${ }^{1}$ Despite advances in multimodality treatment, long-term survival is only seen in $10-20 \%$ of patients due to the advanced stage at presentation. ${ }^{2}$ Surgical resection remains the only potentially curative therapy for patients with iCCA; however, only $30-60 \%$ of patients are candidates for surgical resection due to locally advanced (large tumors with either hepatic inflow or outflow involvement) or metastatic disease, underlying chronic liver disease, or frailty. ${ }^{3}$ In patients undergoing surgical resection, the 5-year survival is $20-40 \%$, and median survival is 25 months. $^{3}$

In patients with unresectable disease, median survival is $12-15$ months with a 5 -year survival of $5-10 \%$, ${ }^{4,5}$ with chemotherapy based on a combination of gemcitabine and platinum salts. ${ }^{6}$ There is no established standard treatment for patients with locally advanced biliary cancers. The role of chemotherapy or radiotherapy is considered mainly a palliative in unresectable iCCA. Neoadjuvant therapy (NAT) with a view to downstage has gained popularity in the last decade in the management of hepatobiliary and pancreatic cancers, particularly the latter in patients with borderline resectable and locally advanced (LA) cancers. Pooled analysis by Suker et al. ${ }^{7}$ in a systematic review demonstrated NAT with FOLFIRINOX for locally advanced pancreatic cancers had a median overall survival (OS) ranging between 10 and 33 months and resection rates of up to $43 \%$. In resectable oesophageal cancers, NAT has

Received: September 13, 2019; Revised: November 2, 2019; Accepted: November 14, 2019

Comesponding author: Bobby VM Dasari

Department of Hepatobiliary and Pancreatic Surgery, Queen Elizabeth Hospital, Edgbaston, Birmingham, B15 2TH, UK Tel: +00447727621372, E-mail: bobby.dasari@yahoo.com

Copyright (C) 2020 by The Korean Association of Hepato-Biliary-Pancreatic Surgery

This is an Open Access article distributed under the terms of the Creative Commons Attribution Non-Commercial License (http://creativecommons.org/ licenses/by-nc/4.0) which permits unrestricted non-commercial use, distribution, and reproduction in any medium, provided the original work is properly cited. Annals of Hepato-Biliary-Pancreatic Surgery - pISSN: 2508-5778 - elSSN: 2508-5859 
been demonstrated standard treatment through high-quality randomized controlled trials. ${ }^{8}$ A recent network metaanalysis further demonstrated neoadjuvant CRT (chemoradiotherapy) followed by surgery to be the most effective strategy in improving long-term survival of resectable oesophageal cancers. ${ }^{9}$

To date, there is limited consensus for advocating NAT for patients with iCCA to further improve survival and surgical outcomes. For the purpose of the study, this was considered downstaging treatment given to patients with non-metastatic iCCA as NAT, although the intention might have been palliation given the clinical practice during the study period. Hence, the aim of this review is to determine the oncological and surgical outcomes of patients receiving NAT for borderline iCCA.

\section{METHODS}

\section{Search strategy}

A systematic search of PubMed, EMBASE and the Cochrane Library databases were conducted. The search terms used were 'intrahepatic cholangiocarcinoma' or 'cholangiocarcinoma', and 'neoadjuvant therapy or 'neoadjuvant radiotherapy' or 'neoadjuvant chemotherapy' or 'chemotherapy' individually or in combination. Search terms used for this review are presented as shown in Supplementary Table 1. The 'related articles' function was used to broaden the search, and all citations were considered for relevance. A manual search of reference lists in recent reviews and eligible studies was also undertaken.

\section{Inclusion and exclusion criteria}

Inclusion criteria were: (1) studies reporting the use of NAT (by any modality) in human subjects with non-metastatic locally advanced iCCA; (2) published in the English language. Exclusion criteria were: (1) conference abstracts, review articles, and case reports ( $<5$ patients); (2) publications with mixed populations where the outcomes of patients with cancers at another site could not be separated from those of patients with intrahepatic cholangiocarcinoma. After excluding duplicates, two authors (SK, BD) independently reviewed the titles and abstracts of studies identified by the literature search. Where a study was considered to be potentially relevant to the research question a full copy of the publication was ob- tained for further review. The references of all included studies were hand-searched in order to identify other potentially relevant studies. Any areas of disagreement between the two primary researchers were resolved through discussion. The intention of the NAT in this review might have been palliative treatment when offered to patients but patients have then progressed to surgical pathways where response has been noted. It is therefore important to note the differences in the terminology used in this review to the terms used in standard clinical practice. Patients who progressed to surgery following down staging treatment are considered to have had surgery following NAT, patients who received treatment but not surgery had palliative chemotherapy, and those who did not receive any treatment (no NAT group) were managed by best supportive care.

\section{Data extraction}

Two researchers (SK, BD) independently extracted data on study characteristics, patient demographics, definitions of borderline resection, modality and regimes of NAT, response to and the clinical benefit with NAT, progression to surgery and postoperative outcomes such as overall mortality and morbidity rates such as bile leak, liver failure, where reported.

\section{Study outcomes}

The primary outcome measure was OS in patients receiving NAT with or without subsequent surgical resection. Response to chemotherapy, where reported, was graded as complete response (CR), partial response (PR), stable disease (SD), and progressive disease (PD). Secondary outcome measures were rates of overall resection rates and margin-negative (R0) resections, overall complications (Grade I-V) and major complications ( $>$ Grade III) reported according to Clavien-Dindo classification, surgery-specific complications (bile leak, intra-abdominal collections), and response to chemotherapy. A pooled analysis of the data was performed to assess the study outcomes and a comparative survival analysis was performed. 


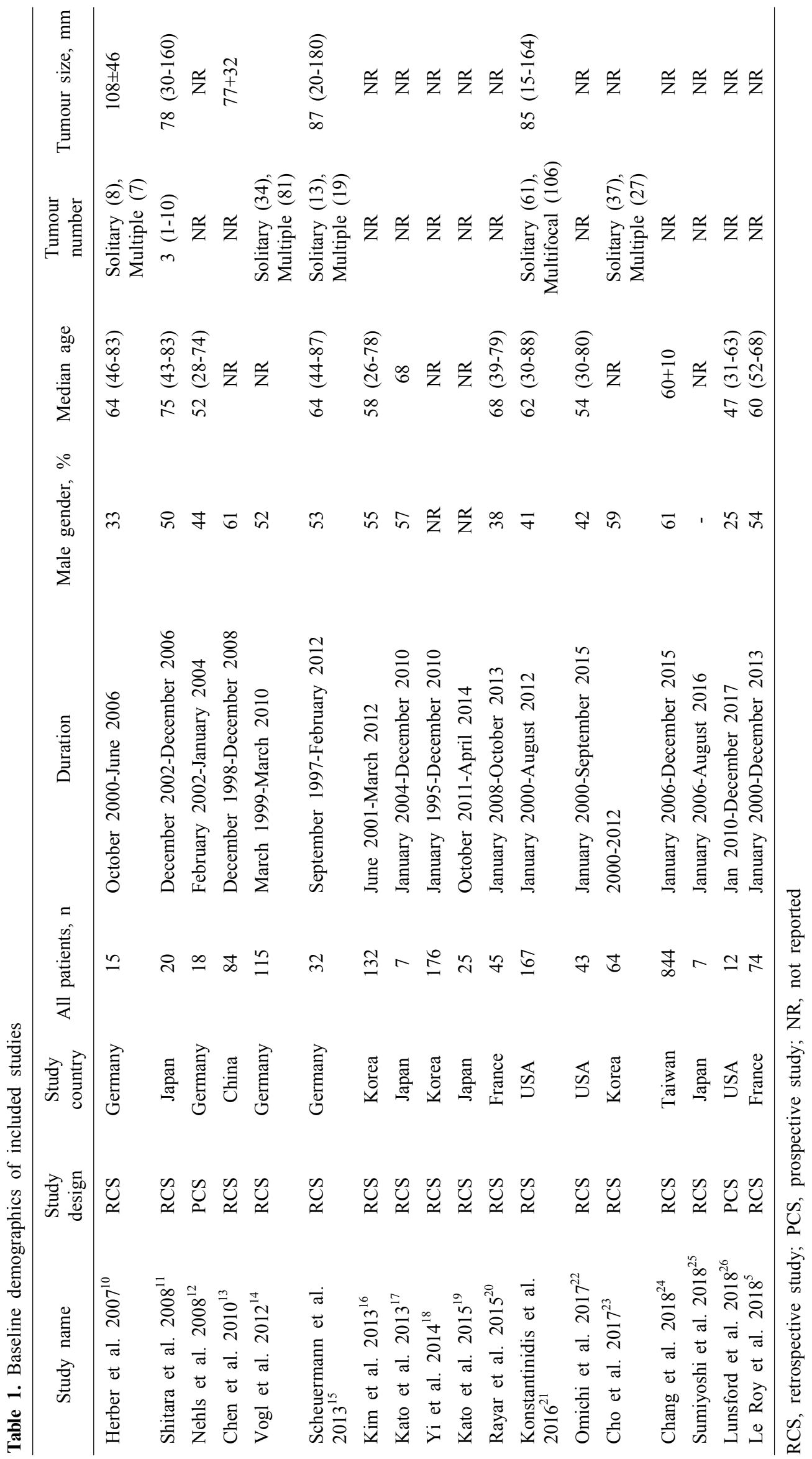




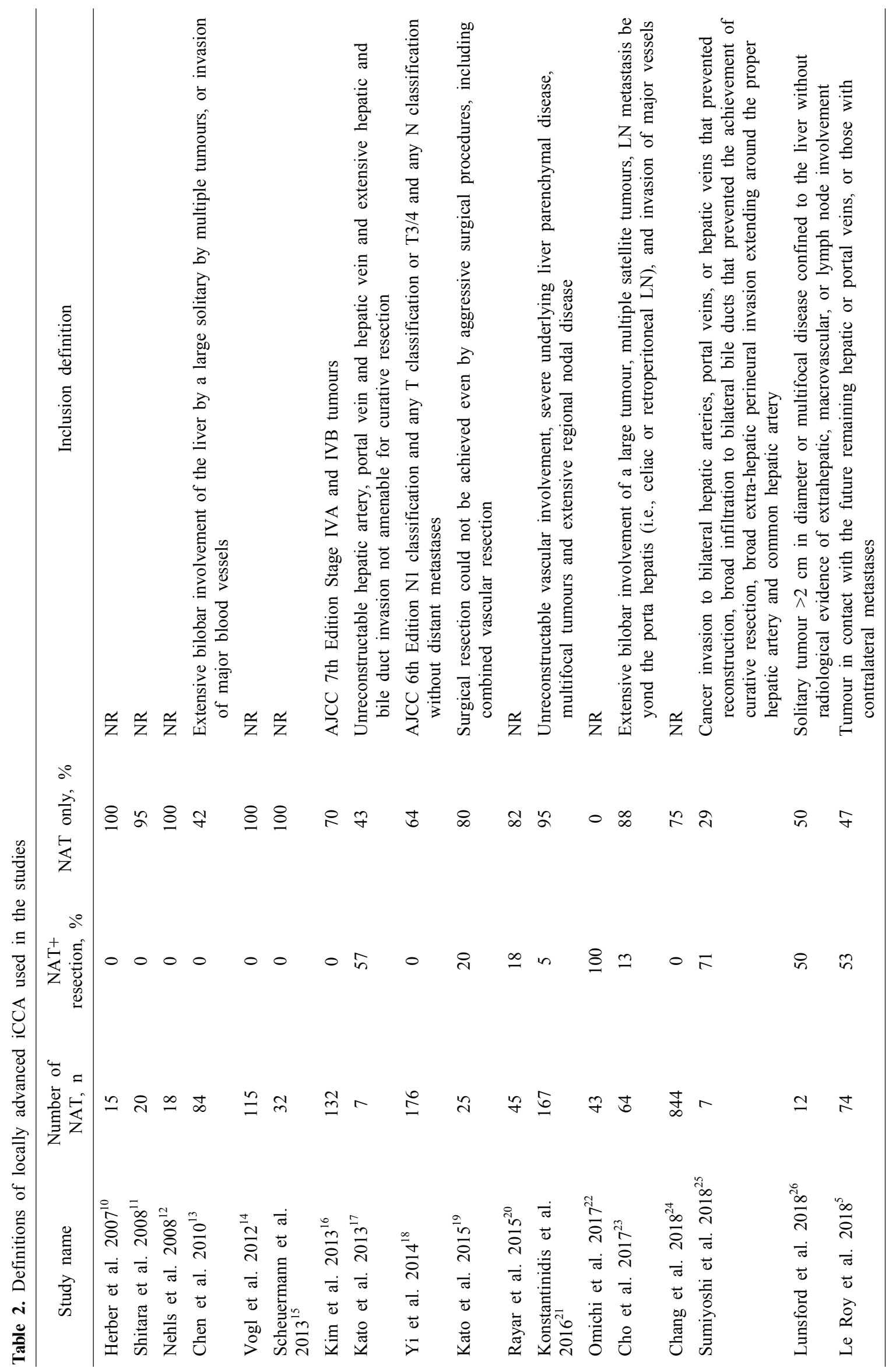




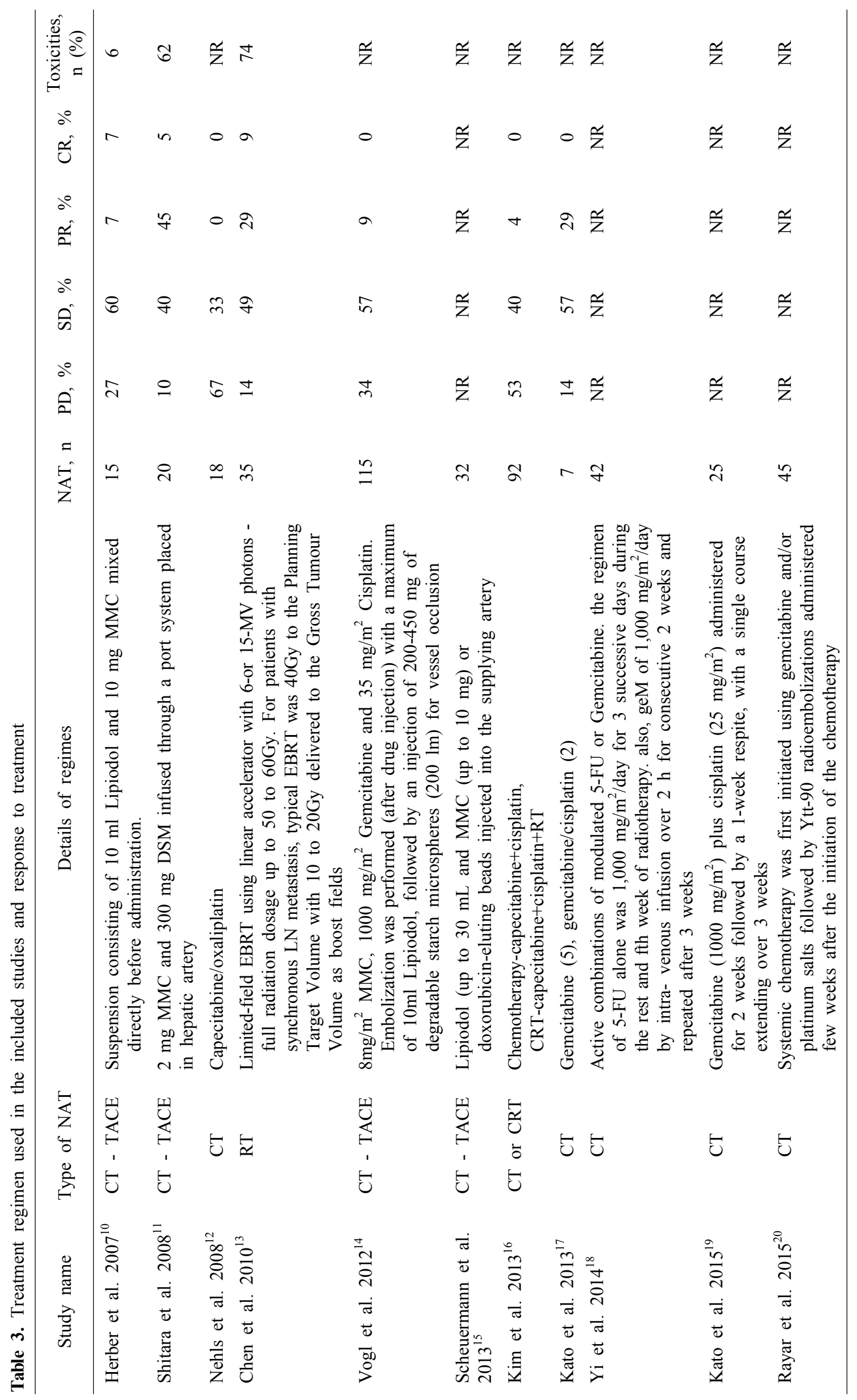




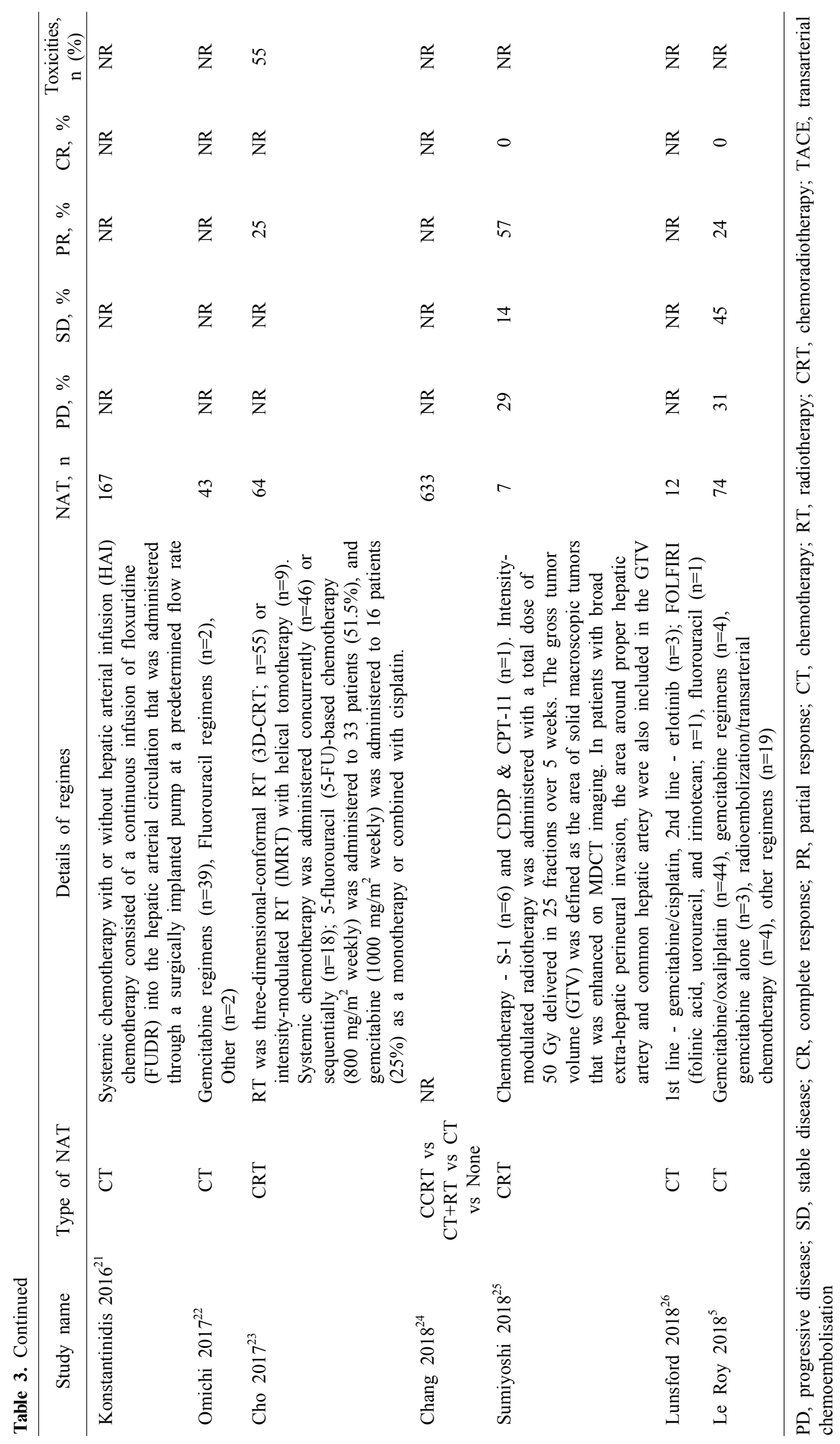




\section{RESULTS}

\section{Study characteristics}

The literature search identified 18 cohort studies, including 1880 patients with locally advanced iCCA, of which two were prospective cohort studies. Baseline demographics of the included studies are presented in Table 1. Study quality was assessed using NewCastle Ottawa system. Ten studies provided the definitions for locally advanced or inoperable iCCA, and these are detailed in Table 2 .

\section{Chemotherapy and radiotherapy regimes}

NAT regimes and tumor responses are presented in Table 3. Eleven studies reported the use of neoadjuvant chemotherapy, of which four ${ }^{10,11,14,15}$ reported the use of transarterial chemoembolization (TACE) with mitomycin $\mathrm{C}$ (MMC). One study ${ }^{14}$ used gemcitabine and cisplatin in addition to MMC for TACE. Eight studies $5,12,17-20,22,26$ evaluated combinations of chemotherapy (gemcitabine/capecitabine and platinum-based regimes). Of the remaining studies, one reported use of $\mathrm{RT}^{13}{ }^{13}$ three reported use of chemoradiotherapy (CRT), ${ }^{16,23,25}$ and one compared concurrent CRT, chemotherapy and RT, chemotherapy, and no treatment. ${ }^{24}$

\section{Response rates and the definitions of response}

Eleven studies reported the criteria used to assess tumour response [RECIST $(\mathrm{n}=9),{ }^{10,11,14,16,17,19,21,23,25}$ mRECIST $(n=1){ }^{5}$ and WHO $\left.(n=1)\right] .^{12}$ Nine studies ${ }^{5,10-14,16,17,25} \quad(n=383$ patients) provided response rates as the presence of progressive disease (PD), stable disease (SD), partial response (PR), and complete response (CR). The rates of PD were $36 \%$, ranging from 10 to $67 \%$. The Clinical Benefit Rate $(\mathrm{CBR}=\mathrm{CR}+\mathrm{PR}+\mathrm{SD})$ was noted in $64 \%$ (244/383) of the patients going for NAT, ranging from 33 to $90 \%$.

\section{Resectability rates}

Of the nine studies ${ }^{5,10-14,16,17,25}$ (383 patients) where response rates were provided, 64\% (244/383) showed a clinical benefit for NAT. None of the studies provided the number of patients who were explored for but failed to proceed to resection following NAT. Of the studies ${ }^{5,17,19-22,25,26}$ where post-NAT resection rates were reported, 135/354 patients (38\%) underwent resections. Of these, $60 \%$ of the patients had R0 resections (82/135 patients), 40\% were reported to have R1 resection, and R2 resection was performed in $1 / 135$ patients.

\section{Overall survival}

The OS of the entire cohort was 14 months (range, 7-18 months). OS was significantly longer in patients receiving NAT with resection (median: 29 months; range: 18-37 months) compared to NAT alone (median: 12 months; range: 5-43 months) or no NAT (median: 8 months; range: 5-11 months) $(p<0.001)$ for locally advanced iCCA. In patients receiving chemotherapy only, the OS of the entire cohort was 18 months (range, 5-20 months). OS was significantly longer in patients receiving NAT with resection (median, 36 months; range, 18-37 months) compared to NAT alone (median, 12 months; range, 5-43 months, $p=0.02$ ). In patients receiving CRT only, the OS of the entire cohort was 12 months (range, 9-15 months). OS was longer in patients receiving NAT with resection (median, 21 months; range, 18-24 months) compared to NAT alone (median: 11 months; range: 9-43 months, $p=0.8$ ) (Table 4).

\section{Postoperative outcomes}

Overall post-surgical complications were reported in eight studies. ${ }^{5,17,19-22,25,26}$ The overall rate of postoperative mortality was $6 \%(8 / 135$ patients) on pooled analysis. The rate of major complications was $15.5 \%$ (21/135 patients). Bile leaks (four patients), post-hepatectomy liver failure (four patients), intraabdominal collections (four patients), ascites (three patients), post-operative bleeding (one patient), pleural effusion (one patient), and acute kidney injury (one patient) were the reported complications (Table $5)$.

\section{DISCUSSION}

Over the last decade, the long-term survival of patients diagnosed with iCCA has been relatively poor, with a small proportion of patients undergoing curative surgical resection and a median survival of 20-30 months in the resected group. ${ }^{3}$ NAT for iCCA is an appealing option for suitable candidates to select the patient with less aggressive tumor biology, downstage the disease, increase 
Table 4. Overall survival among the patients in the all the treatment pathways

\begin{tabular}{|c|c|c|c|c|c|}
\hline Study name & All patients, $\mathrm{n}$ & $\begin{array}{c}\text { OS entire cohort, } \\
\text { months }\end{array}$ & $\begin{array}{l}\text { OS NAT }+ \\
\text { resection, months }\end{array}$ & $\begin{array}{l}\text { OS NAT only, } \\
\text { months }\end{array}$ & $\begin{array}{c}\text { OS no NAT, } \\
\text { months }\end{array}$ \\
\hline Herber et al. $2007^{10}$ & 15 & $21(9-33)$ & - & $21(9-33)$ & NR \\
\hline Shitara et al. $2008^{11}$ & 20 & 14 & - & 14 & NR \\
\hline Nehls et al. $2008^{12}$ & 18 & NR & - & NR & NA \\
\hline Chen et al. $2010^{13}$ & 84 & $7 \pm 1$ & - & $10 \pm 1$ & $5 \pm 1$ \\
\hline Vogl et al. $2012^{14}$ & 115 & 13 & - & 13 & NA \\
\hline Scheuermann et al. $2013^{15}$ & 32 & NR & - & 11 & NR \\
\hline Kim et al. $2013^{16}$ & 132 & 9 & - & 9 & NR \\
\hline Kato et al. $2013^{17}$ & 7 & 13 & 29 & 13 & NA \\
\hline Yi et al. $2014^{18}$ & 176 & NR & - & $43(34-51)$ & $11(7-16)$ \\
\hline Kato et al. $2015^{19}$ & 25 & NR & NR & NR & NR \\
\hline Rayar et al. $2015^{20}$ & 45 & NR & $16(4-41)$ & NR & NR \\
\hline Konstantinidis et al. $2016^{21}$ & 167 & $20(1-120)$ & $37(10-92)$ & NR & NR \\
\hline Omichi et al. $2017^{22}$ & 43 & NR & NR & NA & NA \\
\hline Cho et al. $2017^{23}$ & 64 & NR & NR & NR & NR \\
\hline Chang et al. $2018^{24}$ & 844 & NR & - & NR & NR \\
\hline Sumiyoshi et al. $2018^{25}$ & 7 & NR & NR & NR & NR \\
\hline Lunsford et al. $2018^{26}$ & 12 & NR & 36 & NR & NR \\
\hline Le Roy et al. $2018^{5}$ & 74 & 18 & 36 & 11 & NA \\
\hline
\end{tabular}

Table 5. Post-operative outcomes following resection surgery, where reported

\begin{tabular}{|c|c|c|c|c|c|c|}
\hline & $\begin{array}{c}\text { Numbers } \\
\text { received NAT }\end{array}$ & $\begin{array}{l}\text { Numbers } \\
\text { proceeded to } \\
\text { resection }\end{array}$ & R0 resection & $\mathrm{R} 1$ resection & $\begin{array}{l}\text { Post-op } \\
\text { mortality }\end{array}$ & Major morbidity \\
\hline Kato et al. $2015^{19}$ & 39 & 10 & 7 & 3 & - & - \\
\hline Kato et al. $2013^{17}$ & 22 & 8 & 4 & 4 & - & - \\
\hline Rayar et al. $2015^{20}$ & 45 & 10 & 10 & 0 & 2 & 3 \\
\hline Konstantinidis et al. $2016^{21}$ & 104 & 8 & 5 & 3 & 2 & \\
\hline Sumiyoshi et al. $2018^{25}$ & 15 & 11 & 9 & 2 & - & 3 \\
\hline Omichi et al. $2017^{22}$ & 43 & 43 & 30 & 13 & - & 5 \\
\hline Lunsford et al. $2018^{26}$ & 12 & 6 & 5 & 1 & - & 1 \\
\hline Le Roy et al. $2018^{5}$ & 74 & 39 & 12 & 27 & 4 & 9 \\
\hline Total & 354 & $135(38.1 \%)$ & $82(60.7 \%)$ & $53(39.2 \%)$ & $8(6 \%)$ & $21(15.5 \%)$ \\
\hline
\end{tabular}

the resectability rates, and improve the OS in the locally advanced iCCA. NAT therapy in iCCA lags behind other gastrointestinal cancers, such as oesophageal and pancreatic cancers, where this approach has shown significant improvement in long term-survival and increased the number of ongoing clinical trials. ${ }^{7,9}$ The present review highlights that current evidence for NAT in iCCA is limited to cohort studies, specifically retrospective case series. This review further highlights that NAT followed by resection has a superior survival rate than patients receiving NAT alone or no surgery in the group of patients deemed unresectable because of locally advanced disease. Le Roy et al. ${ }^{5}$ reported no significant difference in the patients who had primarily resectable and downstaged un- resectable lesions in terms of postoperative complications, but despite the higher $\mathrm{R} 1$ resection rates $(p=0.004)$, the OS was similar (HR 1.23, 0.77-1.97; $p=0.391$ ) between the two groups. The current review does not differentiate the outcomes of $\mathrm{R} 1$ and $\mathrm{R} 0$ resections but together the OS was significantly better in the cohort that proceeded for surgery following downstaging NAT.

iCCAs are usually peripherally located, away from the hilum and are usually dealt with by anatomical, non-anatomical resections based on the position of the lesion. When the lesions are larger or located centrally, the inflow or (more often) the outflow of the liver could be infiltrated. Such vascular or biliary involvement on the contralateral side of resection are usually considered the 
limiting factor for upfront resection. ${ }^{25}$ The group of patients with iCCA that is not suitable for surgical resections are usually put through a palliative pathway or best supportive care. Factors such as micro or macro vascular invasion, presence of lymph node metastases, and presence of satellite nodules represent poor pathological prognostic factors. The influence of pathological factors on their outcomes is not clear from this review. However, this study has shown a variable median OS in the group of patients receiving NAT (with or without curative resection) with an encouraging survival benefit. There is a lack of a definition for locally advanced iCCA, which might reflect the lack of consensus about the patients suitable for NAT and type of treatment in the context of multimodal treatment options (Table 2). We propose that the HPB surgical community should aim to obtain consensus for the definition of borderline resectability and selection of iCCA patients for NAT. In this group of patients, NAT might be able to increase resectability rates, with acceptable morbidity, mortality, and prolonged survival.

The response rates of iCCA to chemotherapy are variable and limited. Currently, there is no standard treatment for locally advanced cholangiocarcinoma, either in a NAT or palliative set up. The ABC-02 trial compared doublet therapy with gemcitabine and cisplatin to gemcitabine as a single agent in 410 patients with locally advanced or metastatic biliary tract cancer. ${ }^{6}$ After a median follow-up of 8.2 months, the combination group had a significantly improved OS (11.7 vs. 8.1 months). Similar results with combination chemotherapy were also reported by studies with a $70 \%$ response rate or stable disease and OS of up to 15 months. ${ }^{27}$ The results of $\mathrm{ABC} 06$ trial for 2nd line chemotherapy with FOLFOX regime are awaited but the unpublished results are promising. ${ }^{28}$ Other treatment options, such as chemoradiotherapy, TACE, and external beam RT, have also been associated with longer progression-free survival and OS than chemotherapy alone in a palliative setting for patients with unresectable advanced iCCA. ${ }^{29}$ Shitara et al. ${ }^{11}$ reported a 50\% response rate and a median survival of 14.1 months with hepatic arterial infusion chemotherapy for unresectable iCCA. The review also reflects the variations in the NAT regimes used by the included studies, although the majority of studies used a gemcitabine-based chemotherapy regime and only one study used a standardized regime (gemcitabine/oxaliplatin) in all patients. Other limitations to this study include significant heterogeneity of the included studies with no clear definitions of locally advanced iCCA. Not all of the reported studies reported the survival data, and well-defined post-operative complications, limiting the quality of the meta-analysis to reliably analyze the impact of NAT in patients with and without surgical resection. However, the current study provides the base to plan future studies that would be of useful in the management of this group of patients.

In conclusion, although the evidence to support the benefits of NAT for iCCA is limited, the data from this review is very promising in improving the outcomes of patients with iCCA. International efforts are required to standardize the definitions and treatment regimens targeting locally advanced iCCA through randomized controlled trials.

\section{ORCID}

Sivesh Kamarajah: https://orcid.org/0000-0002-2748-0011

Francesco Giovinazzo: https://orcid.org/0000-0002-33929292

Keith J. Roberts: https://orcid.org/0000-0003-1799-9829

Pankaj Punia: https://orcid.org/0000-0002-4578-6428

Robert P. Sutcliffe: https://orcid.org/0000-0002-1881-7655

Ravi Marudanayagam: https://orcid.org/0000-0002-06403535

Nikolaos Chatzizacharias: https://orcid.org/0000-00024864-189X

John Isaac: https://orcid.org/0000-0002-7946-1277

Darius F. Mirza: https://orcid.org/0000-0002-7531-9089

Paolo Muiesan: https://orcid.org/0000-0002-7389-6691

Bobby VM Dasari: https://orcid.org/0000-0003-2375-1141

\section{AUTHOR CONTRIBUTIONS}

Conceptualization: BD.

Data curation: SK, FG.

Formal analysis: BD, SK, FG, KJR, RPS, PP, RM, NC, JI, DFM, PM.

Methodology: BD, SK.

Project administration: BD, KJR, PP, PM.

Visualization: BD, DFM, RPS, RM.

Writing - original draft: BD, SK. 
Writing - review \&amp; editing: BD, FG, KJR, RPS, PP, RM, NC, JI, DFM, PM.

\section{REFERENCES}

1. Yao KJ, Jabbour S, Parekh N, Lin Y, Moss RA. Increasing mortality in the United States from cholangiocarcinoma: an analysis of the National Center for Health Statistics Database. BMC Gastroenterol 2016;16:117.

2. Kamarajah SK. Evaluation of the AJCC 8th edition staging system for pathologically versus clinically staged intrahepatic cholangiocarcinoma (iCCA): a time to revisit a dogma? A Surveillance, Epidemiology, and End Results (SEER) analysis. J Gastrointest Cancer 2019;50:392-399.

3. Endo I, Gonen M, Yopp AC, Dalal KM, Zhou Q, Klimstra D, et al. Intrahepatic cholangiocarcinoma: rising frequency, improved survival, and determinants of outcome after resection. Ann Surg 2008;248:84-96.

4. Nathan H, Pawlik TM. Staging of intrahepatic cholangiocarcinoma. Curr Opin Gastroenterol 2010;26:269-273.

5. Le Roy B, Gelli M, Pittau G, Allard MA, Pereira B, Serji B, et al. Neoadjuvant chemotherapy for initially unresectable intrahepatic cholangiocarcinoma. Br J Surg 2018;105:839-847.

6. Valle J, Wasan H, Palmer DH, Cunningham D, Anthoney A, Maraveyas A, et al.; ABC-02 Trial Investigators. Cisplatin plus gemcitabine versus gemcitabine for biliary tract cancer. N Engl J Med 2010;362:1273-1281.

7. Suker M, Beumer BR, Sadot E, Marthey L, Faris JE, Mellon EA, et al. FOLFIRINOX for locally advanced pancreatic cancer: a systematic review and patient-level meta analysis. Lancet Oncol 2016;17:801-810.

8. Burmeister BH, Smithers BM, Gebski V, Fitzgerald L, Simes RJ, Devitt P, et al; Trans-Tasman Radiation Oncology Group; Australasian Gastro-Intestinal Trials Group. Surgery alone versus chemoradiotherapy followed by surgery for resectable cancer of the oesophagus: a randomised controlled phase III trial. Lancet Oncol 2005;6:659-668.

9. Pasquali S, Yim G, Vohra RS, Mocellin S, Nyanhongo D, Marriott $\mathrm{P}$, et al. Survival after neoadjuvant and adjuvant treatments compared to surgery alone for resectable esophageal carcinoma: a network meta-analysis. Ann Surg 2017;265:481-491.

10. Herber S, Otto G, Schneider J, Manzl N, Kummer I, Kanzler $\mathrm{S}$, et al. Transarterial chemoembolization (TACE) for inoperable intrahepatic cholangiocarcinoma. Cardiovasc Intervent Radiol 2007;30:1156-1165

11. Shitara K, Ikami I, Munakata M, Muto O, Sakata Y. Hepatic arterial infusion of mitomycin $\mathrm{C}$ with degradable starch microspheres for unresectable intrahepatic cholangiocarcinoma. Clin Oncol (R Coll Radiol) 2008;20:241-246.

12. Nehls O, Oettle H, Hartmann JT, Hofheinz RD, Hass HG, Horger MS, et al. Capecitabine plus oxaliplatin as first-line treatment in patients with advanced biliary system adenocarcinoma: a prospective multicentre phase II trial. Br J Cancer 2008;98: 309-315.

13. Chen YX, Zeng ZC, Tang ZY, Fan J, Zhou J, Jiang W, et al. Determining the role of external beam radiotherapy in unresectable intrahepatic cholangiocarcinoma: a retrospective analysis of 84 patients. BMC Cancer 2010;10:492.

14. Vogl TJ, Naguib NN, Nour-Eldin NE, Bechstein WO, Zeuzem $\mathrm{S}$, Trojan $\mathrm{J}$, et al. Transarterial chemoembolization in the treatment of patients with unresectable cholangiocarcinoma: results and prognostic factors governing treatment success. Int $\mathrm{J}$ Cancer
2012;131:733-740.

15. Scheuermann U, Kaths JM, Heise M, Pitton MB, Weinmann A, Hoppe-Lotichius $\mathrm{M}$, et al. Comparison of resection and transarterial chemoembolisation in the treatment of advanced intrahepatic cholangiocarcinoma--a single-center experience. Eur J Surg Oncol 2013;39:593-600.

16. Kim YI, Park JW, Kim BH, Woo SM, Kim TH, Koh YH, et al. Outcomes of concurrent chemoradiotherapy versus chemotherapy alone for advanced-stage unresectable intrahepatic cholangiocarcinoma. Radiat Oncol 2013;8:292.

17. Kato A, Shimizu H, Ohtsuka M, Yoshidome H, Yoshitomi H, Furukawa K, et al. Surgical resection after downsizing chemotherapy for initially unresectable locally advanced biliary tract cancer: a retrospective single-center study. Ann Surg Oncol 2013;20:318-324.

18. Yi SW, Kang DR, Kim KS, Park MS, Seong J, Park JY, et al. Efficacy of concurrent chemoradiotherapy with 5-fluorouracil or gemcitabine in locally advanced biliary tract cancer. Cancer Chemother Pharmacol 2014;73:191-198.

19. Kato A, Shimizu H, Ohtsuka M, Yoshitomi H, Furukawa K, Takayashiki $\mathrm{T}$, et al. Downsizing chemotherapy for initially unresectable locally advanced biliary tract cancer patients treated with gemcitabine plus cisplatin combination therapy followed by radical surgery. Ann Surg Oncol 2015;22 Suppl 3:S1093-S1099.

20. Rayar M, Sulpice L, Edeline J, Garin E, Levi Sandri GB, Meunier B, et al. Intra-arterial yttrium-90 radioembolization combined with systemic chemotherapy is a promising method for downstaging unresectable huge intrahepatic cholangiocarcinoma to surgical treatment. Ann Surg Oncol 2015;22:3102-3108.

21. Konstantinidis IT, Groot Koerkamp B, Do RK, Gönen M, Fong Y, Allen PJ, et al. Unresectable intrahepatic cholangiocarcinoma: systemic plus hepatic arterial infusion chemotherapy is associated with longer survival in comparison with systemic chemotherapy alone. Cancer 2016;122:758-765.

22. Omichi K, Cloyd JM, Yamashita S, Tzeng CD, Conrad C, Chun YS, et al. Neutrophil-to-lymphocyte ratio predicts prognosis after neoadjuvant chemotherapy and resection of intrahepatic cholangiocarcinoma. Surgery 2017;162:752-765.

23. Cho Y, Kim TH, Seong J. Improved oncologic outcome with chemoradiotherapy followed by surgery in unresectable intrahepatic cholangiocarcinoma. Strahlenther Onkol 2017;193:620629.

24. Chang WW, Hsiao PK, Qin L, Chang CL, Chow JM, Wu SY. Treatment outcomes for unresectable intrahepatic cholangiocarcinoma: nationwide, population-based, cohort study based on propensity score matching with the Mahalanobis metric. Radiother Oncol 2018;129:284-292.

25. Sumiyoshi T, Shima Y, Okabayashi T, Negoro Y, Shimada Y, Iwata $\mathrm{J}$, et al. Chemoradiotherapy for initially unresectable locally advanced cholangiocarcinoma. World J Surg 2018;42:29102918.

26. Lunsford KE, Javle M, Heyne K, Shroff RT, Abdel-Wahab R, Gupta N, et al.; Methodist-MD Anderson Joint Cholangiocarcinoma Collaborative Committee (MMAJCCC). Liver transplantation for locally advanced intrahepatic cholangiocarcinoma treated with neoadjuvant therapy: a prospective case-series. Lancet Gastroenterol Hepatol 2018;3:337-348.

27. Eckmann KR, Patel DK, Landgraf A, Slade JH, Lin E, Kaur H, et al. Chemotherapy outcomes for the treatment of unresectable intrahepatic and hilar cholangiocarcinoma: a retrospective analysis. Gastrointest Cancer Res 2011;4:155-160.

28. Lamarca A, Palmer DH, Wasan HS, Ross PJ, Ma YT, Arora A, et al. ABC-06 | a randomised phase III, multi-centre, open-label study of active symptom control (ASC) alone or ASC with ox- 
aliplatin / 5-FU chemotherapy (ASC+mFOLFOX) for patients (pts) with locally advanced / metastatic biliary tract cancers (ABC) previously-treated with cisplatin/gemcitabine (CisGem) chemotherapy. J Clin Oncol 2019;37(Suppl 15):4003.
29. Currie BM, Soulen MC. Decision making: intra-arterial therapies for cholangiocarcinoma-TACE and TARE. Semin Intervent Radiol 2017;34:92-100. 